\title{
Ações voltadas para o tabagismo: análise de sua implementação na Atenção Primária à Saúde
}

\author{
Actions geared to tobacco control: \\ a review of their implementation in Primary Health Care
}

Leonardo Henriques Portes ${ }^{1}$

Estela Marcia Saraiva Campos ${ }^{2}$

Maria Teresa Bustamante Teixeira ${ }^{2}$

Rosângela Caetano ${ }^{3}$

Luiz Cláudio Ribeiro ${ }^{4}$

${ }^{1}$ Escola Nacional de Saúde Pública. Fiocruz. R.

Leopoldo Bulhões 1480, Manguinhos. 21.041-210 Rio de Janeiro RJ Brasil. leo.portes@yahoo.com.br ${ }^{2}$ Faculdade de Medicina, Universidade Federal de Juiz de Fora.

${ }^{3}$ Instituto de Medicina Social, Universidade do Estado do Rio de Janeiro. ${ }^{4}$ Departamento de Estatística, Universidade Federal de Juiz de Fora.
Abstract This survey examined the status of the implementation of policies for tobacco control in a medium-sized municipality through an assessment of actions recommended by the National Tobacco Control Program (NTCP) promoted by Primary Health Care (PHC) units. The coordinator of the local NTCP and professionals from 44 PHC units of Juiz de Fora in the state of Minas Gerais were interviewed, between May and July 2011. It was observed that the actions of treatment and health promotion were being duly executed, the highlight being the training of professionals to implement treatment in PHC. In 40.9\%, there is the provision of treatment for smokers, and in $88.6 \%$ the actions are concentrated in activities in waiting rooms, groups and individual consultations. Limitations identified are probably not restricted to the municipality, such as inadequate structuring of units, high turnover, the varying degree of involvement of professionals in implementing the service and also the lack of mechanisms to enable them to implement and give continuity to care among their other responsibilities. The major challenge for tobacco control is to conduct intersectoral actions and in primary health care. The results can serve for the formulation of strategic health actions in other parts of the country.

Key words Primary Health Care, National Tobacco Control Program, Smoking
Resumo A pesquisa analisou a situação de implementação das políticas voltadas para o controle do tabagismo em município de médio porte através da verificação das ações preconizadas pelo Programa Nacional de Controle do Tabagismo (PNCT) desenvolvidas em Unidades de Atenção Primária à Saúde (UAPS). Foram entrevistados o coordenador municipal do PNCT e profissionais das 44 UAPS de Juiz de Fora (MG), entre maio e julho de 2011. Observou-se que as ações de tratamento e de promoção da saúde vêm sendo executadas, tendo destaque a capacitação de profissionais para implementação do tratamento nas UAPS. Em 40,9\% das UAPS, há a oferta do tratamento para o fumante e, em 88,6\%, as ações concentram-se em atividades de salas de espera, grupos e consultas individuais. Provavelmente não se restringem ao município a inadequada estruturação das unidades, a alta rotatividade de trabalhadores, o grau variado de comprometimento dos profissionais em implementarem o atendimento e a carência de mecanismos que lhes permitam iniciar e dar continuidade aos atendimentos em meio às demais atribuições que possuem. O grande desafio para o controle do tabagismo é realizar as ações intersetoriais e na atenção primária. Os resultados podem servir para a formulação de ações estratégicas de saúde em outras localidades do país.

Palavras-chave Atenção Primária à Saúde, Programa nacional de controle do tabagismo, Tabagismo 


\section{Introdução}

A fumaça do tabaco é extremamente nociva à saúde. A duração e o nível de exposição à fumaça do tabaco estão diretamente relacionados com o risco e a severidade de muitas consequências adversas à saúde. Em publicação de 2010, que considerou também a exposição passiva ao tabaco, o Centers for Disease Control and Prevention (CDC) sintetizou uma longa lista de consequências e doenças associadas pelo tabagismo. Entre elas estão diversos tipos de neoplasia e doenças respiratórias, cardiovasculares e ósseas ${ }^{1}$.

No mundo, o tabagismo está associado a cerca de 70\% dos casos de câncer de pulmão, 42\% das doenças respiratórias crônicas e $10 \%$ das doenças cardiovasculares² ${ }^{2}$ Além disso, é responsável por impactantes prejuízos sociais, sanitários, econômicos e ambientais ${ }^{3}$.

Estudo de Wünsch Filho et al. ${ }^{4}$, de 2010, concluiu que os recentes inquéritos populacionais realizados no Brasil indicam avanços no controle do uso do tabaco no país, sendo que a prevalência de tabagismo da população apresenta tendência decrescente no tempo em todas as faixas etárias. Vale mencionar que dados do VIGITEL Brasil 2011 - Vigilância de Fatores de Risco e Proteção para Doenças Crônicas por Inquérito Telefônico — revelaram que o percentual de fumantes, entre os homens, correspondia a $18,1 \%$ e, entre as mulheres, a $12 \%$. Os homens, em compensação, estão deixando mais o cigarro: $25 \%$ se declararam ex-fumantes, em comparação a 19\% das mulheres. O número de fumantes acima de 18 anos de idade no Brasil caiu de $15,1 \%$, em 2010, para $14,8 \%$ em $2011^{5}$; a queda é ainda mais expressiva se considerarmos os números do primeiro estudo VIGITEL de 2006, que apontava percentual de $16 \%{ }^{6}$.

Parte desta evolução pode ser atribuída ao Programa Nacional de Controle do Tabagismo (PNCT), que, desde 1989, desenvolve um conjunto diversificado de ações que visam à redução da prevalência de fumantes? .

A Atenção Primária à Saúde (APS) configura-se como cenário oportuno para a execução das ações de controle do tabagismo, uma vez que se caracteriza por:

"um conjunto de ações de saúde, no âmbito individual e coletivo, que abrange a promoção e a proteção da saúde, a prevenção de agravos, o diagnóstico, o tratamento, a reabilitação, a redução de danos e a manutenção da saúde com o objetivo de desenvolver uma atenção integral que impacte na situação de saúde e autonomia das pessoas e nos determinantes e condicionantes de saúde das coletividades"8.

Segundo Starfield ${ }^{9}$, a APS organiza e racionaliza todos os recursos, tanto básicos quanto especializados, direcionados para a promoção, a manutenção e a melhora da saúde. O seu fortalecimento com ênfase nas ações de promoção da saúde permite a melhoria do status desta, a redução de iniquidades e a redução de custos $^{10}$. Assim, os serviços fragmentados necessitam de estratégias inovadoras que permitam a execução de ações preventivas na comunidade e o seu funcionamento de forma coordenada e abrangente ${ }^{11}$.

O compromisso em ampliar o acesso à abordagem e ao tratamento do tabagismo para a rede de APS e de média complexidade do Sistema Único de Saúde (SUS) foi garantido com a Portaria $\mathrm{n}^{\circ} 1035$ de 31 de maio de 2004, do Ministério da Saúde (MS) ${ }^{12}$. Em 13 de agosto do mesmo ano, o MS publicou a Portaria no 442 a qual apresentou como anexos o Plano para Implantação da Abordagem e Tratamento do Tabagismo na rede SUS e o Protocolo Clínico e Diretrizes Terapêuticas - Dependência à Nicotina. O primeiro apresenta informações detalhadas sobre a capacitação de profissionais de saúde, credenciamento de unidades de saúde, cadastramento de usuários, garantia de referência e contrarreferência e fornecimento dos medicamentos utilizados no tratamento da dependência à nicotina.

Já o protocolo clínico sintetiza como deve ser a abordagem do fumante para a cessação de fumar, devendo ter como eixo central intervenções cognitivas e o treinamento de habilidades comportamentais, visando à cessação e à prevenção de recaída. O apoio medicamentoso deve ser utilizado apenas em casos específicos, em que os fumantes apresentam grau elevado de dependência à nicotina ${ }^{13}$.

Considerando que a APS tem como fundamento possibilitar o acesso universal e contínuo a serviços de saúde resolutivos e de qualidade e constitui-se como a principal porta de entrada da Rede de Atenção à Saúde ${ }^{8}$ a oferta de ações de controle do tabagismo torna-se prioritária neste nível de atenção.

Para a melhor qualidade do serviço prestado na atenção básica se configuram como importantes aspectos a capacitação dos profissionais de saúde como ação estratégica para a consolidação do SUS ${ }^{14} \mathrm{e}$ a promoção da atenção integral à saú$\mathrm{de}^{15}$, a adequação da estrutura física das unidades de atenção primária à saúde (UAPS) ${ }^{16} \mathrm{e}$ a articulação intersetorial, uma vez que a saúde é inseparável do desenvolvimento econômico-social ${ }^{17}$. 
No que tange à legislação brasileira referente ao controle do tabagismo, são aspectos importantes a regulação da atividade econômica do setor tabagista, o alerta sobre os perigos da nicotina e a fixação dos limites para seu consumo ${ }^{18}$. "Leis locais em municípios e estados representativos (como Rio de Janeiro e São Paulo) têm tido sucesso em criar espaços 100\% livres de fumo" 19 .

Neste contexto, este estudo teve como objetivos examinar a implementação das ações e diretrizes do PNCT na APS, tomando como cenário um município de médio porte do estado de Minas Gerais, e discutir suas potencialidades e limitações com vistas a contribuir para a difusão e o aprimoramento desse Programa em outras localidades do país.

\section{Métodos}

Segundo Ventura ${ }^{20}$, o estudo de caso apresenta grande utilidade nas pesquisas exploratórias, sendo "recomendável nas fases iniciais de uma investigação sobre temas complexos, para a construção de hipóteses ou reformulação do problema”. Nesse sentido, esta pesquisa tomou como princípio metodológico o estudo de caso de caráter exploratório. O município de Juiz de Fora (MG), que apresentava uma população de 516.247 habitantes no ano de $2010^{21}$, correspondeu ao lócus da pesquisa.

A fim de realizar uma análise do processo de implementação das diretrizes e ações do PNCT no âmbito do município, foi realizada a abordagem qualitativa e adotada a técnica da entrevista. No período de maio a julho de 2011 foram entrevistados 45 sujeitos (coordenador municipal do programa e um profissional de saúde de nível superior de cada uma das 44 UAPS da zona urbana do município). Foram eleitos para a entrevista somente profissionais que foram capacitados para a abordagem e o tratamento do tabagismo e que pudessem fornecer informações sobre o desenvolvimento ou não das ações relacionadas ao tabagismo realizadas pela UAPS onde atuam. No caso das unidades sem profissional capacitado, o próprio gerente local foi o sujeito entrevistado. Todas as 44 UAPS da zona urbana foram eleitas para o estudo por comporem todas as regiões do município, apresentando-se de forma representativa em relação às ações de controle do tabagismo na APS local.

Segundo Duarte 22 , "categorias de análise podem ser eleitas pelo pesquisador antes da realização das entrevistas... a partir de referências teóri- co/conceituais". Dessa forma, as entrevistas foram orientadas por três instrumentos, que objetivaram detectar as diretrizes e as ações previstas no PNCT, compostos por categorias de análise previamente estabelecidas mediante revisão de literatura e documental acerca do Programa.

Um instrumento semiestruturado foi destinado à entrevista do coordenador municipal do PNCT, contendo as seguintes categorias de análise que pudessem evidenciar a situação de implementação do Programa na localidade: Legislação; Prevenção de agravos e Promoção da saúde; e Capacitação de profissionais.

Aos profissionais capacitados para a abordagem e tratamento do tabagismo nas UAPS, foi aplicado um questionário predominantemente estruturado, que tinha como objetivo obter informações estreitamente relacionadas com os anexos da Portaria no 442 do $\mathrm{MS}^{13}$. Por fim, o instrumento utilizado na entrevista com os gerentes das UAPS consistiu em itens mais abrangentes sobre as ações voltadas para o controle do tabagismo. As categorias de análise em ambos os questionários foram as seguintes: Estrutura das UAPS; e Mecanismos de abordagem do usuário.

As entrevistas foram realizadas pelo pesquisador responsável no local e horário de trabalho dos entrevistados. As respostas foram anotadas e gravadas no momento das entrevistas e, posteriormente, seu conteúdo foi transcrito e analisado.

As análises temáticas podem ser um recurso que "encurta o caminho" do pesquisador e o cruzamento do material coletado nas entrevistas com as referências teórico/conceituais implica na construção de um novo texto que promove um "diálogo artificial” entre os dados obtidos, "aproximando respostas semelhantes, complementares ou divergentes de modo a identificar recorrências, concordâncias, contradições, divergências etc" 22 . Nesse sentido, a partir de uma análise temática, os resultados foram organizados conforme as categorias de análise anteriormente mencionadas.

O estudo foi submetido à apreciação do Comitê de Ética em Pesquisa de Seres Humanos da Universidade Federal de Juiz de Fora.

\section{Resultados e discussão}

\section{Características dos sujeitos entrevistados}

O coordenador municipal atua na função desde 2007. Entre os profissionais de saúde, a grande maioria era do sexo feminino (90,9\%), 
de enfermeiros (61,3\%), e com atuação nas suas respectivas UAPS há 9,4 anos em média. Predominaram profissionais que trabalham em UAPS que funcionam com base na Estratégia da Saúde da Família (72,7\%). Quarenta e dois profissionais $(95,4 \%)$ estavam capacitados para a abordagem e tratamento do fumante, sendo entrevistados somente dois gerentes locais correspondentes às UAPS que não apresentavam profissionais submetidos à capacitação para a abordagem e tratamento do fumante.

\section{PNCT em Juiz de Fora}

\section{Legislação}

A implementação do PNCT em Juiz de Fora se iniciou em abril de 2005, quando foi criada a Coordenação Municipal de Controle do Tabagismo, denominada Serviço de Controle, Prevenção e Tratamento do Tabagismo (SECOPTT) ${ }^{23}$.

Na tentativa de implementar o PNCT, foi buscado um conjunto de ações com cunho político e que envolviam o tratamento, a prevenção e a promoção da saúde. Estas ações políticas podem ser evidenciadas pela criação da Lei Municipal no 11.813 de 29/07/2009, que proíbe o fumo em recintos coletivos públicos e privados de Juiz de Fora, assim como nas áreas fechadas de locais de trabalho, onde ocorrer o trânsito, a circulação, a convivência e a permanência de pessoas ${ }^{24}$. Tal iniciativa merece destaque pelo fato de, no período que antecedeu a lei local, ainda estar em vigência a redação original da Lei Nacional $n^{\circ}$ 9.294, de 15 de julho de 1996, que permitia o fumo em área de recintos coletivos "destinada exclusivamente a esse fim, devidamente isolada e com arejamento conveniente" 25 .

O fato de Juiz de Fora ter em vigência uma lei desde 2009, que proíbe o fumo em áreas fechadas de recintos coletivos públicos e privados, evidencia a postura ativa que a gestão municipal de saúde apresenta frente ao controle do tabagismo.

Da mesma forma, também é possível notar avanços em nível federal relacionados aos ambientes livres do tabaco. Em 2011, foi publicada a Lei Nacional no 12.546 , que pró́be o uso de produtos derivados do tabaco inclusive nas áreas que eram antes destinadas exclusivamente aos fumantes ${ }^{26}$.

Prevenção de agravos e Promoção da saúde

O coordenador municipal relatou que as ações de prevenção e promoção são aquelas representadas pelas campanhas nas datas comemorativas de controle do tabagismo (31 de maio e 29 de agosto) e pelas palestras em instituições e empresas, divulgadas por meio de rádio e TV e que conta, em alguns momentos, com parcerias com alunos de graduação da Universidade Federal de Juiz de Fora. Também são programadas campanhas em escolas e salas de espera dos serviços de saúde.

No entanto, não houve grande referência às ações de promoção da saúde que são apontadas pela Política Nacional de Promoção da Saúde ${ }^{27}$ e pelo $\mathrm{PNCT}^{28}$, como o incentivo de ações em ambientes de trabalho capazes de manter um fluxo contínuo de informações sobre o tabagismo e a articulação com o setor educação no sentido de estimular a promoção da saúde no ambiente escolar, respectivamente. A implantação destas ações representa um grande desafio, uma vez que nesse processo, "explicita-se a complexidade da operacionalização da intersetorialidade, pois sua aplicação implica a superação das hierarquias institucionais e das relações de poder existentes entre as corporações profissionais, os setores, as políticas e os segmentos sociais" 29 .

\section{Capacitação de profissionais}

Em relação às ações que envolvem o tratamento do fumante, a sua concretização tem como base a capacitação de profissionais de saúde de todos os níveis de atenção à saúde para o tratamento dos fumantes:

A capacitação dos profissionais é baseada na Portaria $n^{0} 1035$, que visa a facilitar o acesso do fumante ao tratamento e a abordagem cognitivocomportamental dos fumantes [...] A abordagem intensiva é para os de nivel superior, sendo mais estruturada e inclui as sessões em grupo e terapia medicamentosa [...] Há prioridade na capacitação desta abordagem porque permite a oferta do tratamento do fumante com a intenção de descentralizar e implementar o atendimento ao fumante nas unidades básicas de saúde [...] Já a capacitação da abordagem breve/minima é destinada aos profissionais de nivel médio, tendo o foco em motivar o fumante a parar de fumar. (Coordenador municipal)

As capacitações pelo SECOPTT se iniciaram em 2005 e, até o presente, já foram realizadas 1351, tendo sido qualificados 679 profissionais de nível superior e 672 profissionais de nível médio.

Os processos de capacitação dividem-se em dois momentos. Uma parte teórica, realizada em doze horas, aborda, entre outros, o tema tabagismo, os modos de organizar os grupos de tratamento, o controle destes grupos, os instrumentos de avaliação do tratamento e os relatórios, e 
a confecção do projeto em relação à área de abrangência dos profissionais. Além disso, são realizadas quatro horas de atividades práticas, visando ao acompanhamento da implementação, pelo SECOPTT, do primeiro grupo de tratamento nas unidades, com discussão da prática do atendimento e dos instrumentos de controle e avaliação do Instituto Nacional de Câncer.

A partir do relato do coordenador municipal do Programa, torna-se evidente a priorização dos profissionais de saúde que atuam na APS na capacitação para o tratamento do tabagismo. Isto pode ser justificado pelo entendimento da gestão municipal em ter este nível de atenção como eixo coordenador do sistema frente aos demais níveis. Concentrar as ações de tratamento do tabagismo na APS surge como uma possibilidade de obtenção de maior número de usuários beneficiados e consequente maior probabilidade de efetividade na cessação do hábito de fumar.

No entanto, considerando que a capacitação de profissionais com ensino superior é enfatizada pela Portaria $\mathrm{n}^{\mathrm{o}} 442$ do $\mathrm{MS}^{13}$ e o relato do coordenador municipal do Programa que a capacitação para a abordagem intensiva se restringe aos profissionais com ensino superior, a ampliação da capacitação de profissionais de nível médio para a abordagem intensiva poderia representar uma ferramenta a mais para o PNCT. Este argumento é ainda mais relevante se considerado o potencial dos Agentes Comunitários de Saúde (ACS), que se fazem presentes em grande número nas unidades e exercem um papel mediador, manifestado tanto em relação à população, no sentido de facilitar o acesso ao serviço, quanto em relação aos demais profissionais, representando uma importante estratégia para que as propostas dos serviços sejam absorvidas pela comunidade ${ }^{30}$.

Ainda é válido ressaltar outro aspecto que envolve a capacitação dos profissionais para a abordagem ao fumante. O Programa Unidades de Saúde Livres do Cigarro, parte do Programa Saúde e Coerência do PNCT defende as unidades de saúde como vitrine de hábitos de vida saudáveis, devendo ser livres da poluição tabagística e com profissionais que sejam modelo de comportamento.

Assim, preconiza a capacitação de profissionais de saúde para, inicialmente, o apoio à cessação dos funcionários fumantes e, posteriormente, a prestação de atendimento à população ${ }^{28}$. Entretanto, as portarias no 1035 e 442 do MS não enfatizam esta sequência, sendo destacada a ampliação da oferta de tratamento ao usuário que pretende parar de fumar ${ }^{12,13}$. Foram frequentes as citações destes documentos pela coordenação municipal do Programa, sugerindo um maior peso atribuído a eles para a definição do planejamento das ações de controle do tabagismo no município e, assim, um maior foco na prestação de atendimento ao usuário.

\section{Especificidades do PNCT no âmbito da APS}

\section{Estrutura das UAPS}

Não é permitido fumar no interior de nenhuma UAPS. Todas as unidades possuem locais para atendimento individual ao fumante, condição necessária para a garantia de privacidade e espaço para explorar as singularidades de cada indivíduo na sua relação com o tabagismo. Alguns respondentes, contudo, referiram que diversos destes espaços são locais improvisados e pouco adequados para tais atividades, originando dificuldades e prejuízos ao atendimento.

Cerca de 75\% das UAPS (33) possuem espaço para às sessões em grupo dentro da própria unidade e cinco utilizam áreas externas, como sociedades de pró-melhoramento dos bairros, igrejas e escolas. O restante lança mão tanto de espaços internos quanto externos para estas atividades, o que permite aproximação com outras populações que não apenas aquelas que buscam os serviços de saúde.

Diante do exposto, evidencia-se que nem todas as UAPS apresentam estrutura física adequada para a realização do tratamento dos fumantes em grupo. Estrutura deficiente pode contribuir para que haja desestímulo na execução do trabalho, ações ineficientes ou até mesmo a sua não realização. Além disso, compromete profundamente as ações de caráter educativo, resultando em baixa oferta de tais ações nos serviços e consequente focalização nas ações de caráter curativo, de abordagem individual.

A busca pela parceria com estabelecimentos de outros setores demonstrou-se como uma alternativa para muitas unidades que não apresentam espaço adequado para a realização dos grupos de tratamento do tabagismo. É premente avançar para o desenvolvimento de projetos intersetoriais, trazendo de fato o compromisso com ações de promoção da saúde e melhoria da qualidade de vida para a lógica da corresponsabilização, articulando saúde, usuários e outros setores das políticas públicas. No entanto, não é satisfatório que a aproximação com os demais setores ocorra estritamente pelo fato de haver fragilidades na estrutura das UAPS. 
Na perspectiva da APS no âmbito municipal, Giovanella et al. ${ }^{31}$ consideram que a atuação intersetorial configura-se por meio da ação comunitária no território, da articulação na secretaria de saúde e da articulação de políticas municipais. É neste sentido que a parceria com outros setores na própria comunidade pode se apresentar como ferramenta para a criação de uma dinâmica de atenção que resulte em maior adesão e participação ativa dos usuários e profissionais no desenvolvimento das ações de apoio à cessação ao fumo, possibilitando o surgimento de estratégias de ação não limitadas a abordagem do usuário nas UAPS.

\section{Mecanismos de abordagem na APS}

Todo fumante que demonstra interesse em parar de fumar é cadastrado para esta demanda em 34 unidades (77,3\%). Considerando o fato de não ofertarem o tratamento para tabagistas, quatro UAPS não cadastram os fumantes para essas ações e outras quatro não realizam este cadastramento, mas encaminham os fumantes para outras unidades que oferecem o serviço. Dois entrevistados de UAPS distintas relataram nunca ter ocorrido o fato de fumantes procurarem a UAPS para tratamento do tabagismo, o que pode sugerir que talvez não esteja havendo suficiente difusão da informação sobre a disponibilidade da oferta deste serviço entre a população assistida.

É importante ressaltar um desvio das diretrizes nacionais do PNCT acerca do cadastramento dos usuários para o tratamento do tabagismo. Apesar de preconizado que todo fumante que demonstre interesse em parar de fumar deve ser cadastrado na unidade de saúde ${ }^{13}$, o coordenador municipal relatou que a ficha de cadastramento recomendada pelo PNCT deve ser destinada somente aos usuários que comparecem ao segundo encontro nas UAPS. Não cadastrar todos os fumantes no momento em que procuram as unidades segundo a ficha preconizada limita a identificação do perfil dos usuários adscritos em cada unidade, compromete a avaliação e o planejamento das ações destinadas ao fumante e pode levar a uma avaliação errônea do desempenho municipal das ações desenvolvidas.

De todas as 44 UAPS, 18 (40,9\%) oferecem atendimento específico ao fumante segundo o modelo preconizado pelo PNCT. Entre elas, 12 adotam a Estratégia Saúde da Família e seis o modelo assistencial tradicional.

Todas as 18 unidades que oferecem a abordagem cognitivo-comportamental utilizam o material de apoio cedido pelo MS durante as ses- sões em grupo, mas o uso de outros materiais durante as sessões foi frequentemente citado, os quais incluem vídeos educativos, folhetos, material adesivo e cartazes confeccionados pelas próprias unidades ou fornecidos pela prefeitura, governo estadual e a universidade federal do município.

Assim como a abordagem comportamental, todas as 18 UAPS que atendem o fumante segundo o PNCT oferecem tratamento medicamentoso, o que pode estar relacionado à preocupação da coordenação do Programa com a oferta de medicamentos.

É importante refletir, no caso do tabagismo, se a oferta indiscriminada do apoio medicamentoso nos serviços consistirá em uma medida eficaz na cessação do fumo ou se resumirá na oferta do atendimento sem a consideração das particularidades de cada usuário, que certamente não serão atendidas integralmente pela sua restrita medicalização.

Apesar dos medicamentos se destacarem no tratamento do tabagismo, seja pelo controle criterioso da esfera municipal e federal sobre o seu fornecimento, seja pela já conhecida alta demanda da população por este tipo de tratamento ${ }^{32}$, é necessária a sensibilização dos profissionais de saúde sobre a importância do rigor na avaliação da necessidade de sua adoção em complementação à abordagem cognitivo-comportamental e, caso esta necessidade se manifeste, na eleição do tipo a ser adotado.

Ações não específicas para o usuário tabagista são também bastante frequentes nas unidades, tendo sido citadas por 39 entrevistados e apresentam-se sumarizadas na Tabela 1 .

Dentre estas ações, foram muito citadas as atividades de salas de espera, concebidas como espaços onde é feita a sensibilização dos usuários sobre os danos do tabagismo para a saúde e a importância de preveni-los e tratá-los. Muitos entrevistados mencionaram que estes momentos são comumente trabalhados nas datas comemorativas do controle do tabagismo. As atividades em grupo também se apresentaram como oportunidades em que o tema tabagismo é eventualmente abordado. Foram citados os grupos de "hipertensos", de "diabéticos", "desnutridos" entre outros.

A maior prevalência da realização de orientações em sala de espera e atividades em grupo acerca dos riscos do tabagismo pode ser considerada como esperada, uma vez que estes espaços, principalmente as ações em grupo, configuram-se como boas oportunidades para o esclarecimen- 
Tabela 1. Ações não específicas ao tratamento do fumante ofertadas pelas Unidades de Atenção Primária à Saúde da zona urbana de Juiz de Fora/MG.

\begin{tabular}{|c|c|c|c|c|}
\hline \multirow[b]{2}{*}{ Ações } & \multicolumn{2}{|c|}{$\operatorname{Sim}^{*}$} & \multicolumn{2}{|c|}{ Não } \\
\hline & $\mathrm{N}$ & $\%^{* *}$ & $\mathbf{N}$ & $\%$ \\
\hline Salas de espera & 26 & $66,7 \%$ & 13 & $33,3 \%$ \\
\hline Grupos & 22 & $56,4 \%$ & 17 & $43,6 \%$ \\
\hline Consultas na UAPS & 24 & $61,5 \%$ & 15 & $38,5 \%$ \\
\hline Visitas domiciliares & 6 & $15,4 \%$ & 33 & $84,6 \%$ \\
\hline Abordagem em escolas & 3 & $7,7 \%$ & 36 & $92,3 \%$ \\
\hline Abordagem em associações & 1 & $2,6 \%$ & 38 & $97,4 \%$ \\
\hline Busca ativa por fumantes & 1 & $2,6 \%$ & 38 & $97,4 \%$ \\
\hline Divulgação das ações realizadas pela equipe da unidade em eventos científicos & 1 & $2,6 \%$ & 38 & $97,4 \%$ \\
\hline Palestras & 1 & $2,6 \%$ & 38 & $97,4 \%$ \\
\hline
\end{tabular}

"Várias UAPS ofertavam múltiplas atividades inespecíficas; ${ }^{* *}$ Percentuais calculados em função das 39 unidades que relataram ofertar este tipo de ações.

to de informações sobre as doenças, para a troca de conhecimentos e experiências ${ }^{33}$. No entanto, é importante chamar a atenção para a denominação dos grupos citados, que pode sugerir a marcante presença do modelo assistencial tradicional em alguns deles devido à citação de doenças ou acometimentos como suas identificações, como no caso dos grupos de "hipertensos", "diabéticos" e "desnutridos". Não focalizar as patologias como forma de denominação dos grupos tende a valorizar a importância das ações de promoção de saúde ${ }^{34}$.

Foram frequentes menções à abordagem do tema nas consultas individuais cotidianas realizadas nas próprias unidades. Foi notada preocupação dos profissionais em aproveitar estes momentos como uma oportunidade para orientar os usuários fumantes acerca do abandono do hábito de fumar e os não fumantes sobre a importância de não iniciá-lo, alertando sobre os prejuízos do cigarro e sua condição de fator de risco para várias doenças.

Menos frequentes como momentos de abordagem de questões relacionadas ao tabagismo são as visitas domiciliares, estando os diferentes profissionais das unidades envolvidos nestas ações, incluindo os ACS. Uma entrevistada descreveu o que é abordado nestes momentos com maior riqueza de detalhes:

A gente procura, nas visitas, sensibilizar sobre os prejuizos do fumo e buscar alternativas para quem diz que não tem condições em parar de fumar [...] A gente busca estabelecer na casa um lugar para fumar... para não prejudicar o restante da família. (Profissional 13)
Em termos dos espaços fora das unidades, foram mencionadas as atividades nas escolas, mediante formação de grupos de discussão de estudantes e da abordagem das drogas de um modo geral. Uma única UAPS realiza abordagem do controle do tabagismo em associações, destacando-se o trabalho com adolescentes de um projeto social do bairro e com a associação local de moradores de rua. Palestras em clubes e divulgação das ações em eventos científicos mereceram citações pontuais.

A busca ativa de fumantes, realizada pelos ACS a partir de suas visitas domiciliares mensais, parece também ser uma ação infrequente, apenas tendo sido citada por um respondente.

Mesmo havendo a orientação da Política Nacional de Atenção Básica de que o cuidado em saúde deve também ser realizado, quando necessário, no domicílio e nos demais espaços comunitários ${ }^{8}$, ações que extrapolam o âmbito da UAPS são raras. A sobrecarga de trabalho vivenciada por muitos profissionais e as dificuldades na implementação e continuidade de ações intersetoriais podem configurar-se como motivos para tal situação.

\section{Limitações do estudo}

A possibilidade de eventuais conflitos de interesse dos vários sujeitos entrevistados e o fato de que a menção de uma ação estar sendo desenvolvida dentro de uma UAPS não traduzir, necessariamente, como de fato ela está sendo realizada, são elementos que devem ser considerados na apreciação dos resultados. Também merece ser levado em consideração que, embora tenha 
havido o cuidado em se entrevistar um profissional por UAPS, o número de profissionais capacitados e daqueles que efetivamente estão desenvolvendo as ações nos serviços é muito superior.

Cabe ter em mente ainda a impossibilidade de generalização dos resultados obtidos a partir de uma abordagem qualitativa em uma única localidade, embora se acredite que muitas das características e dificuldades aqui apontadas possam estar sendo experimentadas em outros municípios do país, com graus de implementação semelhantes ao PNCT em nível municipal.

\section{Considerações Finais}

De maneira geral, os resultados evidenciaram que apesar da existência de um forte arcabouço organizativo para o controle do tabagismo, se faz presente o desafio das ações de controle do tabagismo se configurarem, de fato, na efetivação do PNCT em nível municipal, desafio este que deve ser enfrentado pelas demais localidades do país que possuem a proposta de combate ao tabagismo.

Pôde-se observar o peso dado às diretrizes nacionais do PNCT, principalmente no que tange às portarias $\mathrm{n}^{\circ} 442^{13} \mathrm{e} 1.035^{12}$ do MS, o que sugere que o foco na capacitação de profissionais para a oferta de atendimento ao fumante não seja uma particularidade do município estudado.

Há de se valorizar a configuração de um cenário que organize a capacitação de profissionais de saúde de forma periódica. Porém, fragilidades devem ser evitadas como a não uniformidade da capacitação dos profissionais pertencentes a uma mesma UAPS, que ameaça os princípios da equidade e da universalidade, além de sobrecarregar os profissionais das equipes Saúde da Família que foram capacitados, caso passem a ofertar atendimento aos usuários não adscritos à sua área de atuação.

A concretização do atendimento ao fumante a partir da capacitação dos profissionais de saúde esbarra em diversas limitações que, provavelmente, não se restringem a Juiz de Fora. Entre estas mereceram destaque a inadequada estruturação das unidades, o grau variado de comprometimento dos profissionais em implementarem o atendimento e a disposição, em segundo plano, da capacitação dos profissionais sem nível superior. Além disso, questões como a alta rotatividade de profissionais nas unidades e a carência de mecanismos que permitam aos profissionais implementarem e darem continuidade aos atendimentos em meio às demais atribuições que possuem, configuram-se como obstáculos ao desafio de efetivar a oferta do atendimento ao fumante de forma contínua e resolutiva.

Como alternativas para a melhoria da oferta do atendimento ao fumante na APS podem ser citadas a articulação da coordenação municipal do PNCT com os demais setores da APS para o planejamento das ações de controle do tabagismo, de modo a serem otimizados os momentos da abordagem do usuário e diminuída a sobrecarga dos profissionais de saúde, assim como a consulta aos próprios profissionais acerca da viabilidade da implementação das ações sugeridas nas capacitações e de estratégias para a superação das dificuldades existentes.

A efetivação do PNCT em nível municipal é um desafio que deve ser encarado pela APS no contexto nacional. O relevante impacto do tabagismo na saúde da população mundial aponta para a importância da superação das limitações e obstáculos que dificultam a real implementação do PNCT na atenção básica, como forma de modificação desta grave situação.

\section{Colaboradores}

LH Portes autor principal participou da concepção, análise e interpretação dos dados; redação do artigo; e da aprovação da versão a ser publicada. LC Ribeiro, SEM Campos, MTB Teixeira e R Caetano participaram da concepção, análise e interpretação dos dados. 


\section{Referências}

1. U.S. Department of Health and Human Services. How Tobacco Smoke Causes Disease: The Biology and Behavioral Basis for Smoking-Attributable Disease: A Report of the Surgeon General. Atlanta, GA: U.S. Department of Health and Human Services, Centers for Disease Control and Prevention, National Center for Chronic Disease Prevention and Health Promotion. Washington: Office on Smoking and Health; 2010.

2. Organização Mundial de Saúde (OMS). Global health risks: mortality and burden of disease attributable to selected major risks. [página na Internet] 2009. [acessado 2011 jan 12]. Disponível em: http:/ /www.who.int/healthinfo/global_burden_disease/ en/index.html

3. Brasil. Ministério das Relações Exteriores. Decreto $n^{\circ}$ 5.658, de 2 de janeiro de 2006. Divisão de Atos Internacionais. Diário Oficial da União 2006; 3 jan. http://www2.mre.gov.br/dai/m_5658_2006.htm. Acesso em: 18 nov. 2010 .

4. Wünsch Filho V, Mirra AP, López RVM, Antunes LF. Tabagismo e câncer no Brasil: evidências e perspectivas. Rev. bras. epidemiol. 2010; 13(2):175-187.

5. Brasil. Ministério da Saúde (MS). Secretaria de Vigilância em Saúde. Secretaria de Gestão Estratégica e Participativa. Vigitel Brasil 2011: vigilância de fatores de risco e proteção para doenças crônicas por inquérito telefônico. Apresentação Vigitel 2011. [página na Internet] Abr. 2012. [acessado 2011 jul 18]. Disponível em: http://bvsms.saude.gov.br/bvs/ palestras/Lancamento_Vigitel_2011_final_ apresenta\%C3\%A7\%C3\%A3o.pdf

6. Brasil. Ministério da Saúde (MS). Secretaria de Vigilância em Saúde. Secretaria de Gestão Estratégica e Participativa. Vigitel Brasil 2006: vigilância de fatores de risco e proteção para doenças crônicas por inquérito telefônico. Brasília: MS; 2007.

7. Cavalcante TM. O controle do tabagismo no Brasil: avanços e desafios. Rev. psiquiatr. clín. 2005; 32(5):283-300.

8. Brasil. Ministério da Saúde (MS). Portaria no 2.488, de 21 de outubro de 2011. Diário Oficial da União 2011; 22 out.

9. Starfield B. Atenção primária: equilíbrio entre necessidades de saúde, serviços e tecnologia. Brasília: Unesco, Ministério da Saúde (MS); 2002.

10. Milstein B, Homer J, Hirsch G. Analyzing national health reform strategies with a dynamic simulation model. Am J Public Health 2010; 100(5):811-819.

11. Onocko-Campos RT, Campos Gastão WS, Ferrer AL, Corrêa CRS, Madureira PR, Gama CP, Dantas DV, Nascimento R. Avaliação de estratégias inovadoras na organização da Atenção Primária à Saúde. Rev Saude Publica 2012; 46(1):43-50.

12. Brasil. Ministério da Saúde (MS). Portaria no 1035. Diário Oficial da União 2007; 31 maio.

13. Brasil. Ministério da Saúde (MS). Portaria no 442. Diário Oficial da União 2004; 13 ago.

14. Melo Marisa LC, Nascimento M A. Treinamento introdutório para enfermeiras dirigentes: possibilidades para gestão do SUS. Rev. bras. enferm. 2003; 56(6):674-677.
15. Torres HC, Amaral MA, Amorim MM, Cyrino AP, Bodstein R. Capacitação de profissionais da atenção primária à saúde para educação em Diabetes Mellitus. Acta Paul Enferm 2010; 23(6):751-756.

16. Silveira DS, Santos IS, Costa JSD. Atenção pré-natal na rede básica: uma avaliação da estrutura e do processo. Cad Saude Publica 2001; 17(1):131-139.

17. Heimann LS, Ibanhes LC, Boaretto RC, Castro IEN, Telesi Júnior E, Cortizo CT, Fausto MCR, Nascimento VB, Kayano J. Atenção primária em saúde: um estudo multidimensional sobre os desafios e potencialidades na Região Metropolitana de São Paulo (SP, Brasil). Cien Saude Colet 2011; 16(6):2877-2888.

18. Ribeiro WC, Julio RS, Curi MA, Nuintin AA. Reflexões sobre a regulamentação jurídica do setor tabagista e saúde pública. Revista de Direito Sanitário 2011; 12(2):59-81.

19. Bialous SA, Presman S, Gigliotti A, Muggli M, Hurt R. A resposta da indústria do tabaco à criação de espaços livres de fumo no Brasil. Rev Panam Salud Pública 2010; 27(4):283-290.

20. Ventura MM. O Estudo de caso como modalidade de pesquisa. Rev SOCERJ. 2007; 20(5):383-386.

21. Instituto Brasileiro de Geografia e Estatística (IBGE). IBGE Cidades. [página na Internet]. [acessado 2011 maio 20]. Disponível em: http://.ibge.gov.br/cidade sat/topwindow.htm?

22. Duarte R. Entrevistas em pesquisa qualitativa. Educar em revista 2004; 24:213-225.

23. Dias HM. Programa municipal de controle do Tabagismo no município de Juiz de Fora: a especificidade do tratamento na atenção básica. 2011 [dissertação]. Juiz de Fora: Universidade Federal de Juiz de Fora; 2012.

24. Prefeitura de Juiz de Fora. Lei no 11.813 , de 29 de julho de 2009. Proíbe o fumo nos recintos coletivos no município de Juiz de Fora e dá outras providências. Projeto n. ${ }^{\circ}$ 068, de autoria do Vereador José Laerte. Secretaria de administração e recursos humanos. Atos do governo. Publicação: 30 de julho de 2009.

25. Brasil. Ministério da Saúde (MS). Lei no 9.294, de 14 de 15 de julho de 1996. Dispõe sobre as restrições ao uso e à propaganda de produtos fumígeros, bebidas alcoólicas, medicamentos, terapias e defensivos agrícolas, nos termos do $\$ 4^{\circ}$ do art. 220 da Constituição Federal. Diário Oficial da União 1996; 16 jul.

26. Brasil. Ministério da Saúde (MS). Lei n. ${ }^{\circ}$ 12.546, de 14 de dezembro de 2011. Institui o Regime Especial de Reintegração de Valores Tributários para as Empresas Exportadoras (Reintegra); dispõe sobre a redução do Imposto sobre Produtos Industrializados (IPI) à indústria automotiva; e dá outras providências. Diário Oficial da União 2011; 15 dez.

27. Brasil. Ministério da Saúde (MS). Secretaria de Vigilância em Saúde. Secretaria de Atenção à Saúde. Política Nacional de Promoção da Saúde. 3a Edição. Brasília: MS; 2010. (Série B. Textos Básicos de Saúde. Série Pactos pela Saúde 2006; v. 7) 
28. Brasil. Ministério da Saúde (MS). Instituto Nacional do Câncer (INCA). Programa Nacional de Controle do Tabagismo e Outros Fatores de Risco: modelo lógico e avaliação. 2a Edição. Rio de Janeiro: INCA, 2003.

29. Silva KL, Rodrigues AT. Ações intersetoriais para promoção da saúde na Estratégia Saúde da Família: experiências, desafios e possibilidades. Rev. bras. Enferm. 2010; 63(5):762-769.

30. Bornstein VJ, Stotz EN. O trabalho dos agentes comunitários de saúde: entre a mediação convencedora e a transformadora. Trab Educ Saúde 2008 Nov2009 Fev; 6(3):457-480.

31. Giovanella L, Mendonça MHM, Almeida PF, Escorel S, Senna MCM, Fausto MCR, Delgado MM, Andrade CLT, Cunha MS, Martins MIC, Teixeira CP. Saúde da família: limites e possibilidades para uma abordagem integral de atenção primária à saúde no Brasil. Cien Saude Colet 2009; 14(3):783-794.

32. Garnelo L, Wright R. Doença, cura e serviços de saúde. Representações, práticas e demandas $\mathrm{Ba}-$ níwa. Cad Saude Publica 2001; 17(2):273-284.

33. Maffacciolli R, Lopes M JM. Os grupos na atenção básica de saúde de Porto Alegre: usos e modos de intervenção terapêutica. Cien Saude Colet 2011; 16(Supl. 1):973-998.

34. Portes LH, Caldas Maria AJ, Paula LT, Freitas MS. Atuação do fisioterapeuta na Atenção Básica à Saúde: uma revisão da literatura brasileira. Rev. APS. 2011; 14(1):111-119.

Artigo apresentado em 17/08/2012

Aprovado em 06/08/2013

Versão final apresentada em 03/12/2013 